\title{
NONTERMINATING EXTENSIONS OF THE SEARS TRANSFORMATION
}

\author{
WENCHANG CHU AND NADIA N. LI
}

\begin{abstract}
By means of two and three term relations of ${ }_{3} \phi_{2}$-series, we investigate the nonterminating ${ }_{4} \phi_{3}$-series. Eight transformation formulaes into double series are established. Four of them are shown to be nonterminating extensions of the Sears transformation.
\end{abstract}

\section{INTRODUCTION AND MOTIVATION}

For the two indeterminates $x$ and $q$, the shifted factorial of $x$ with base $q$ reads as

$(x ; q)_{0}=1$ and $(x ; q)_{n}=(1-x)(1-q x) \cdots\left(1-q^{n-1} x\right)$ for $n \in \mathbb{N}$. When $|q|<1$, we have two well-defined infinite products

$$
(x ; q)_{\infty}=\prod_{k=0}^{\infty}\left(1-q^{k} x\right) \text { and }(x ; q)_{n}=(x ; q)_{\infty} /\left(q^{n} x ; q\right)_{\infty} .
$$

The product and fraction of shifted factorials are abbreviated respectively to

$$
\begin{aligned}
{[\alpha, \beta, \ldots, \gamma ; q]_{n} } & =(\alpha ; q)_{n}(\beta ; q)_{n} \cdots(\gamma ; q)_{n} \\
{\left[\begin{array}{l}
\alpha, \beta, \ldots, \gamma \\
A, B, \ldots, C
\end{array} \mid q\right]_{n} } & =\frac{(\alpha ; q)_{n}(\beta ; q)_{n} \cdots(\gamma ; q)_{n}}{(A ; q)_{n}(B ; q)_{n} \cdots(C ; q)_{n}} .
\end{aligned}
$$

Following Gasper-Rahman [4], the basic hypergeometric series is defined by

$$
{ }_{1+r} \phi_{s}\left[\begin{array}{r}
a_{0}, a_{1}, \ldots, a_{r} \\
b_{1}, \ldots, b_{s}
\end{array} \mid q ; z\right]=\sum_{n=0}^{\infty}\left\{(-1)^{n} q^{\left(\begin{array}{c}
n \\
2
\end{array}\right)}\right\}^{s-r}\left[\begin{array}{c}
a_{0}, a_{1}, \ldots, a_{r} \\
q, b_{1}, \ldots, b_{s}
\end{array} \mid q\right]_{n} z^{n}
$$

where the base $q$ will be restricted to $|q|<1$ for nonterminating $q$-series. Most of the $q$-series results concern the case $r=s$. When the parameters

2010 Mathematics Subject Classification. Primary 33D15; Secondary 05A15.

Key words and phrases. Basic hypergeometric series; The $q$-Pfaff-Saalschütz theorem; The Sears transformation; The $q$-Kampé de Fériet series.

Copyright (c) 2016 by ANUBIH. 
satisfy the condition $q a_{0} a_{1} a_{2} \cdots a_{r}=b_{1} b_{2} \cdots b_{r}$, the series is said to be balanced. There are numerous important summation and transformation formulae in the $q$-series theory. One of them is due to Sears [5] (cf. [4, III-15]), which concerns the terminating balanced ${ }_{4} \phi_{3}$-series subject to the condition $\alpha \beta \gamma=q^{1-n} b c d$ :

$$
{ }_{4} \phi_{3}\left[\begin{array}{c}
q^{-n}, b, c, d \\
\alpha, \beta, \gamma
\end{array} \mid q ; q\right]=\left[\begin{array}{c}
\alpha / c, \gamma / c \\
\alpha, \gamma
\end{array} \mid q\right]_{n}{ }_{4} \phi_{3}\left[\begin{array}{c}
q^{-n}, c, \beta / b, \beta / d \\
\beta, \alpha \beta / b d, \beta \gamma / b d
\end{array} \mid q ; q\right] .
$$

The aim of this paper is to investigate its nonterminating form

$$
{ }_{4} \phi_{3}\left[\begin{array}{c}
b, c, d, e \\
\alpha, \beta, \gamma
\end{array} \mid q ; \frac{\alpha \beta \gamma}{b c d e}\right]=\sum_{n \geq 0}\left[\begin{array}{c}
c, e, b d / \beta \\
q, \alpha, \gamma
\end{array} \mid q\right]_{n}\left(\frac{\alpha \beta \gamma}{b c d e}\right)^{n}\left[\begin{array}{c}
b, d \\
\beta, b d / \beta
\end{array} \mid q\right]_{n} .
$$

Recall the $q$-Pfaff-Saalschütz summation theorem (cf. [1, §8.4] and [4, II-12])

$$
{ }_{3} \phi_{2}\left[\begin{array}{c}
q^{-n}, a, \quad b \\
c, q^{1-n} a b / c \mid
\end{array} \mid q ; q\right]=\left[\begin{array}{c}
c / a, c / b \\
c, c / a b
\end{array} \mid q\right]_{n} .
$$

This can be utilized to rewrite the afore-displayed ${ }_{4} \phi_{3}$-series as

$$
\begin{aligned}
{ }_{4} \phi_{3}\left[\begin{array}{c}
b, c, d, e \\
\alpha, \beta, \gamma
\end{array} \mid q ; \frac{\alpha \beta \gamma}{b c d e}\right] & =\sum_{n \geq 0}\left[\begin{array}{c}
c, e, b d / \beta \\
q, \alpha, \gamma
\end{array} \mid q\right]_{n}\left(\frac{\alpha \beta \gamma}{b c d e}\right)^{n}{ }_{3} \phi_{2}\left[\begin{array}{c}
q^{-n}, \beta / b, \beta / d \\
\beta, q^{1-n} \beta / b d
\end{array} \mid q ; q\right] \\
& =\sum_{n \geq 0}\left[\begin{array}{c}
c, e, b d / \beta \\
q, \alpha, \gamma
\end{array} \mid q\right]_{n}\left(\frac{\alpha \beta \gamma}{b c d e}\right)^{n} \sum_{k=0}^{n}\left[\begin{array}{c}
q^{-n}, \beta / b, \beta / d \\
\beta, q^{1-n} \beta / b d
\end{array} \mid q\right]_{k} q^{k} .
\end{aligned}
$$

Letting $n=k+i$ in the last double sum and then interchanging the summation order, we get the following expression

$$
\begin{aligned}
{ }_{4} \phi_{3}\left[\begin{array}{c}
b, c, d, e \\
\alpha, \beta, \gamma
\end{array} \mid q ; \frac{\alpha \beta \gamma}{b c d e}\right]= & \sum_{k \geq 0}\left[\begin{array}{c}
c, e, \beta / b, \beta / d \\
q, \alpha, \beta, \gamma
\end{array} \mid q\right]_{n}\left(\frac{\alpha \gamma}{c e}\right)^{k} \\
& \times{ }_{3} \phi_{2}\left[\begin{array}{c}
q^{k} c, q^{k} e, b d / \beta \\
q^{k} \alpha, q^{k} \gamma
\end{array} \mid q ; \frac{\alpha \beta \gamma}{b c d e}\right] .
\end{aligned}
$$

This will be our starting point for the subsequent development. By means of the $q$-Kummer-Thomae-Whipple transformation and the Hall transformation (cf. Gasper-Rahman [4, III-9 \& 10]), we shall derive four double series expressions for the ${ }_{4} \phi_{3}$-series given in (2) in the next section. Then in the third section, further four transformation formulae will be established by utilizing the two three-term transformations (cf. Gasper-Rahman [4, III-33 $\& 34])$. Finally, the paper will end with a discussion of some known results that are contained as particular cases of the main theorems proved in this paper. 


\section{TRAnsformations Into DOUble SERIES}

Based on the double sum expression displayed in (4), we shall derive four transformation theorems in this section. The main tools are the $q$-KummerThomae-Whipple transformation (cf. Gasper-Rahman [4, III-9])

$$
{ }_{3} \phi_{2}\left[\begin{array}{c}
b, c, d \\
\alpha, \gamma
\end{array} \mid q ; \frac{\alpha \gamma}{b c d}\right]=\left[\begin{array}{c}
\alpha / d, \alpha \gamma / b c \\
\alpha, \alpha \gamma / b c d
\end{array} \mid q\right]_{\infty}{ }_{3} \phi_{2}\left[\begin{array}{c}
d, \gamma / b, \gamma / c \mid \\
\gamma, \alpha \gamma / b c
\end{array} \mid q ; \frac{\alpha}{d}\right]
$$

and the Hall transformation (cf. Gasper-Rahman [4, III-10])

$$
{ }_{3} \phi_{2}\left[\begin{array}{c}
b, c, d \\
\alpha, \gamma
\end{array} \mid q ; \frac{\alpha \gamma}{b c d}\right]=\left[\begin{array}{c}
b, \alpha \gamma / b d, \alpha \gamma / b c \\
\alpha, \gamma, \alpha \gamma / b c d
\end{array} \mid q\right]_{\infty}{ }_{3} \phi_{2}\left[\begin{array}{c}
\alpha / b, \gamma / b, \alpha \gamma / b c d \\
\alpha \gamma / b d, \alpha \gamma / b c
\end{array} \mid q ; b\right] .
$$

2.1. According to (5), we can reformulate the ${ }_{3} \phi_{2}$-series displayed in (4) as

$$
\begin{aligned}
{ }_{3} \phi_{2}\left[\begin{array}{c}
q^{k} c, q^{k} e, b d / \beta \\
q^{k} \alpha, q^{k} \gamma
\end{array} \mid q ; \frac{\alpha \beta \gamma}{b c d e}\right]= & {\left[\begin{array}{c}
\alpha \gamma / c e, q^{k} \alpha \beta / b d \\
q^{k} \alpha, \alpha \beta \gamma / b c d e
\end{array} \mid q\right]_{\infty} } \\
& \times{ }_{3} \phi_{2}\left[\begin{array}{c}
b d / \beta, \gamma / c, \gamma / e \\
\alpha \gamma / c e, q^{k} \gamma
\end{array} \mid q ; \frac{q^{k} \alpha \beta}{b d}\right] .
\end{aligned}
$$

Substituting the last expression into (4), we get the first transformation formula.

Theorem 1 (Double sum expression).

$$
\begin{aligned}
& { }_{4} \phi_{3}\left[\begin{array}{c}
b, c, d, e \\
\alpha, \beta, \gamma
\end{array} \mid q ; \frac{\alpha \beta \gamma}{b c d e}\right]=\left[\begin{array}{c}
\alpha \beta / b d, \alpha \gamma / c e \\
\alpha, \alpha \beta \gamma / b c d e
\end{array} \mid q\right]_{\infty} \\
& \times \sum_{k, i \geq 0} \frac{q^{k i}}{(\gamma ; q)_{k+i}}\left[\begin{array}{c}
c, e, \beta / b, \beta / d \\
q, \beta, \alpha \beta / b d
\end{array} \mid q\right]_{k}\left(\frac{\alpha \gamma}{c e}\right)^{k}\left[\begin{array}{c}
b d / \beta, \gamma / c, \gamma / e \\
q, \alpha \gamma / c e
\end{array} \mid q\right]_{i}\left(\frac{\alpha \beta}{b d}\right)^{i} .
\end{aligned}
$$

When $d=1$, the last theorem gives, under the replacement $b \rightarrow b \beta$, the following closed formula for the double series, which is equivalent to Gasper's one (cf. Gasper-Rahman [4, P300]).

Corollary 2 (Summation formula of double series).

$$
\left[\begin{array}{c}
\alpha, \alpha \gamma / b c e \\
\alpha / b, \alpha \gamma / c e
\end{array} \mid q\right]_{\infty}=\sum_{k, i \geq 0} \frac{q^{k i}}{(\gamma ; q)_{k+i}}\left[\begin{array}{c}
c, e, 1 / b \\
q, \alpha / b
\end{array} \mid q\right]_{k}\left(\frac{\alpha \gamma}{c e}\right)^{k}\left[\begin{array}{c}
b, \gamma / c, \gamma / e \\
q, \alpha \gamma / c e
\end{array} \mid q\right]_{i}\left(\frac{\alpha}{b}\right)^{i}
$$

The last formula may be considered as a double series extension of the well-known $q$-Gauss summation theorem (cf. Bailey[1, §8.4] and GasperRahman [4, II-8]) because for $\gamma=e$, it reduces equivalently to the following

$$
{ }_{2} \phi_{1}\left[\begin{array}{r}
a, b \\
c \mid q ; \frac{c}{a b}
\end{array}\right]=\left[\begin{array}{c}
c / a, c / b \\
c, c / a b
\end{array} \mid q\right]_{\infty} \quad \text { where } \quad|c / a b|<1 .
$$


2.2. Alternatively, applying (5) to the ${ }_{3} \phi_{2}$-series displayed in (4)

$$
\begin{aligned}
{ }_{3} \phi_{2}\left[\begin{array}{c}
q^{k} c, q^{k} e, b d / \beta \\
q^{k} \alpha, q^{k} \gamma
\end{array} \mid q ; \frac{\alpha \beta \gamma}{b c d e}\right] & =\left[\begin{array}{c}
q^{k} c, \alpha \gamma / c e, q^{k} \alpha \beta \gamma / b c d \\
q^{k} \alpha, q^{k} \gamma, \alpha \beta \gamma / b c d e
\end{array} \mid q\right]_{\infty} \\
& \times{ }_{3} \phi_{2}\left[\begin{array}{c}
\alpha / c, \gamma / c, \alpha \beta \gamma / b c d e \\
\alpha \gamma / c e, q^{k} \alpha \beta \gamma / b c d
\end{array} \mid q ; q^{k} c\right]
\end{aligned}
$$

we derive from (4) the second transformation formula.

Theorem 3 (Double sum expression).

$$
\begin{aligned}
& { }_{4} \phi_{3}\left[\begin{array}{c}
b, c, d, e \\
\alpha, \beta, \gamma
\end{array} \mid q ; \frac{\alpha \beta \gamma}{b c d e}\right]=\left[\begin{array}{c}
c, \alpha \gamma / c e, \alpha \beta \gamma / b c d \\
\alpha, \gamma, \alpha \beta \gamma / b c d e
\end{array} \mid q\right]_{\infty} \\
& \times \sum_{k, i \geq 0} \frac{q^{k i} c^{i}}{(\alpha \beta \gamma / b c d ; q)_{k+i}}\left[\begin{array}{c}
e, \beta / b, \beta / d \\
q, \beta
\end{array} \mid q\right]_{k}\left(\frac{\alpha \gamma}{c e}\right)^{k}\left[\begin{array}{c}
\alpha / c, \gamma / c, \alpha \beta \gamma / b c d e \\
q, \alpha \gamma / c e
\end{array} \mid q\right]_{i} .
\end{aligned}
$$

Letting $\beta=e=b d$ in the last theorem, we get the following interesting reduction formula for the $q$-Appell like $\Phi^{(3)}$-series (cf. Gasper-Rahman [4, $\S 10.2])$.

Corollary 4 (Reduction formula of double series).

$$
\begin{aligned}
{ }_{3} \phi_{2}\left[\begin{array}{c}
b, c, d \\
\alpha, \gamma
\end{array} \mid q ; \frac{\alpha \gamma}{b c d}\right] & {\left[\begin{array}{c}
\alpha, \gamma \\
c, \alpha \gamma / c \mid
\end{array} \mid q\right]_{\infty} } \\
& =\sum_{k, i \geq 0} \frac{q^{k i} c^{i}}{(\alpha \gamma / c ; q)_{k+i}}\left[\begin{array}{c}
b, d \\
q
\end{array} \mid q\right]_{k}\left(\frac{\alpha \gamma}{b c d}\right)^{k}\left[\begin{array}{c}
\alpha / c, \gamma / c \\
q
\end{array} \mid q\right]_{i}
\end{aligned}
$$

This identity extends again the $q$-Gauss summation theorem (7), which corresponds, in fact, to the particular case $d=1$ of the formula displayed in the last corollary.

2.3. By means of $(6)$, the ${ }_{3} \phi_{2}$-series in (4) can be reformulated as

$$
\begin{aligned}
& { }_{3} \phi_{2}\left[\begin{array}{c}
q^{k} c, q^{k} e, b d / \beta \\
q^{k} \alpha, q^{k} \gamma
\end{array} \mid q ; \frac{\alpha \beta \gamma}{b c d e}\right] \\
& =\left[\begin{array}{c}
\alpha / e, q^{k} \alpha \beta \gamma / b c d \\
q^{k} \alpha, \alpha \beta \gamma / b c d e
\end{array} \mid q\right]{ }_{3} \phi_{2}\left[\begin{array}{c}
q^{k} e, \gamma / c, q^{k} \beta \gamma / b d \\
q^{k} \gamma, q^{k} \alpha \beta \gamma / b c d
\end{array} \mid q ; \frac{\alpha}{e}\right] .
\end{aligned}
$$

Substituting this expression into (4), we get the third transformation formula.

Theorem 5 (Double sum expression).

$$
{ }_{4} \phi_{3}\left[\begin{array}{c}
b, c, d, e \\
\alpha, \beta, \gamma
\end{array} \mid q ; \frac{\alpha \beta \gamma}{b c d e}\right]=\left[\begin{array}{c}
\alpha / e, \alpha \beta \gamma / b c d \\
\alpha, \alpha \beta \gamma / b c d e
\end{array} \mid q\right]_{\infty}
$$




$$
\times \sum_{k, i \geq 0}\left[\begin{array}{c}
e, \beta \gamma / b d \\
\gamma, \alpha \beta \gamma / b c d
\end{array} \mid q\right]_{k+i}\left(\frac{\alpha \gamma}{c e}\right)^{k}\left[\begin{array}{c}
c, \beta / b, \beta / d \\
q, \beta, \beta \gamma / b d
\end{array} \mid q\right]_{k} \frac{(\gamma / c ; q)_{i}}{(q ; q)_{i}}\left(\frac{\alpha}{e}\right)^{i} .
$$

Letting $\beta=0$ in the last theorem, we find another interesting summation

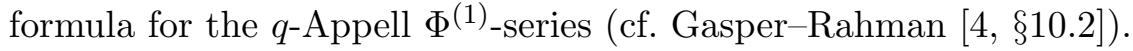

Corollary 6 (Summation formula of double series).

$$
\frac{(\alpha ; q)_{\infty}}{(\alpha / e ; q)_{\infty}}=\sum_{k, i \geq 0} \frac{(e ; q)_{k+i}}{(\gamma ; q)_{k+i}} \frac{(c ; q)_{k}}{(q ; q)_{k}}\left(\frac{\alpha \gamma}{c e}\right)^{k} \frac{(\gamma / c ; q)_{i}}{(q ; q)_{i}}\left(\frac{\alpha}{e}\right)^{i} .
$$

When $\gamma=0$, this identity recovers the $q$-binomial series (cf. $[1, \S 8.2]$ and $[4, \mathrm{II}-3])$

$$
{ }_{1} \phi_{0}\left[\begin{array}{c}
a \\
-
\end{array} \mid q ; x\right]=\frac{(a x ; q)_{\infty}}{(x ; q)_{\infty}} \quad \text { where } \quad|x|<1 .
$$

2.4. Analogously, by utilizing (6), we can restate ${ }_{3} \phi_{2}$-series in (4) as

$$
\begin{aligned}
{ }_{3} \phi_{2}\left[\begin{array}{c}
q^{k} c, q^{k} e, b d / \beta \mid q ; \frac{\alpha \beta \gamma}{b c d e} \\
q^{k} \alpha, q^{k} \gamma
\end{array}\right. & =\left[\begin{array}{c}
b d / \beta, q^{k} \alpha \beta \gamma / b c d, q^{k} \alpha \beta \gamma / b d e \\
q^{k} \alpha, q^{k} \gamma, \alpha \beta \gamma / b c d
\end{array}\right]_{\infty} \\
& \times{ }_{3} \phi_{2}\left[\begin{array}{c}
\alpha \beta \gamma / b c d e, q^{k} \alpha \beta / b d, q^{k} \beta \gamma / b d \\
q^{k} \alpha \beta \gamma / b c d, q^{k} \alpha \beta \gamma / b d e
\end{array} \mid q ; \frac{b d}{\beta}\right] .
\end{aligned}
$$

This leads (4) to the fourth transformation formula.

Theorem 7 (Double sum expression).

$$
\begin{aligned}
{ }_{4} \phi_{3}\left[\begin{array}{c}
b, c, d, e \\
\alpha, \beta, \gamma
\end{array} \mid q ; \frac{\alpha \beta \gamma}{b c d e}\right] & =\left[\begin{array}{c}
b d / \beta, \alpha \beta \gamma / b c d, \alpha \beta \gamma / b d e \\
\alpha, \gamma, \alpha \beta \gamma / b c d e
\end{array} \mid q\right]_{\infty} \sum_{k, i \geq 0}\left[\begin{array}{c}
\frac{\alpha \beta}{b d}, \frac{\beta \gamma}{b d} \\
\frac{\alpha \beta \gamma}{b c d}, \frac{\alpha \beta \gamma}{b d e} \mid q
\end{array}\right]_{k+i} \\
& \times\left[\begin{array}{c}
c, e, \beta / b, \beta / d \\
q, \beta, \frac{\alpha \beta}{b d}, \frac{\beta \gamma}{b d}
\end{array} \mid q\right]_{k}\left(\frac{\alpha \gamma}{c e}\right)^{k} \frac{(\alpha \beta \gamma / b c d e ; q)_{i}}{(q ; q)_{i}}\left(\frac{b d}{\beta}\right)^{i} .
\end{aligned}
$$

Letting $d=1$ and then replacing $b$ by $b \beta$ in the last theorem, we obtain the following interesting summation formula.

Corollary 8 (Summation formula of double series).

$$
\begin{aligned}
& {\left[\begin{array}{c}
\alpha, \gamma, \alpha \gamma / b c e \\
b, \alpha \gamma / b c, \alpha \gamma / b e
\end{array} \mid q\right]_{\infty}=\sum_{k, i \geq 0}\left[\begin{array}{c}
\alpha / b, \gamma / b \\
\alpha \gamma / b c, \alpha \gamma / b e
\end{array} \mid q\right]_{k+i} } \\
& \times\left[\left.\begin{array}{c}
c, e, 1 / b \\
q, \alpha / b, \gamma / b
\end{array}\right|_{k}\right]_{k}\left(\frac{\alpha \gamma}{c e}\right)^{k} \frac{(\alpha \gamma / b c e ; q)_{i}}{(q ; q)_{i}} b^{i}
\end{aligned}
$$

When $c=1$, this identity recovers again the $q$-Gauss summation theorem (7). 


\section{TWO-TERMS TRANSFORMATIONS}

In this section, four transformation theorems involving two double sums will be established by employing the following two three-term transformation formulae (cf. Gasper-Rahman [4, III-33 \& 34]):

$$
\begin{gathered}
{ }_{3} \phi_{2}\left[\begin{array}{c}
b, c, d \\
\alpha, \gamma
\end{array} \mid q ; \frac{\alpha \gamma}{a b c}\right]=\left[\begin{array}{c}
\alpha / b, \alpha / c \\
\alpha, \alpha / b c
\end{array} \mid q\right]_{\infty}{ }_{3} \phi_{2}\left[\begin{array}{c}
b, c, \gamma / d \\
\gamma, q b c / \alpha
\end{array} \mid q ; q\right] \\
+\left[\begin{array}{c}
b, c, \gamma / d, \alpha \gamma / b c \\
\alpha, \gamma, b c / \alpha, \alpha \gamma / b c d
\end{array} \mid q\right]_{\infty}{ }_{3} \phi_{2}\left[\begin{array}{c}
\alpha / b, \alpha / c, \alpha \gamma / b c d \\
\alpha \gamma / b c, q \alpha / b c
\end{array} \mid q ; q\right] \\
{ }_{3} \phi_{2}\left[\begin{array}{c}
b, c, d \\
\alpha, \gamma
\end{array} \mid q ; \frac{\alpha \gamma}{b c d}\right]=\left[\begin{array}{c}
\alpha / b, \alpha / c, q c / d, q / \gamma \\
\alpha, q c / \gamma, q / d, \alpha / b c
\end{array} \mid q\right]_{\infty}{ }_{3} \phi_{2}\left[\begin{array}{c}
c, \gamma / d, q c / \alpha \\
q c / d, q b c / \alpha
\end{array} \mid q ; \frac{q b}{\gamma}\right] \\
-\left[\begin{array}{c}
q / \gamma, q \alpha / \gamma, b, c, \gamma / d, \alpha \gamma / b c q, q^{2} b c / \alpha \gamma \\
\gamma / q, \alpha, q b / \gamma, q c / \gamma, q / d, \alpha / b c, q b c / \alpha
\end{array} \mid q\right]_{\infty}{ }_{3} \phi_{2}\left[\begin{array}{c}
\frac{q b}{\gamma}, \frac{q c}{\gamma}, \frac{q d}{\gamma} \\
\frac{q^{2}}{\gamma}, \frac{q \alpha}{\gamma} \mid q ; \frac{\alpha \gamma}{b c d}
\end{array}\right] .
\end{gathered}
$$

3.1. According to (9), we can reformulate the ${ }_{3} \phi_{2}$-series in (4) as

$$
\begin{aligned}
& { }_{3} \phi_{2}\left[\begin{array}{c}
q^{k} c, q^{k} e, b d / \beta \\
q^{k} \alpha, q^{k} \gamma
\end{array} \mid q ; \frac{\alpha \beta \gamma}{b c d e}\right]=\left[\begin{array}{c}
\alpha / e, q^{k} \alpha \beta / b d \\
q^{k} \alpha, \alpha \beta / b d e
\end{array} \mid q\right]_{\infty}{ }_{3} \phi_{2}\left[\begin{array}{c}
q^{k} e, \gamma / c, b d / \beta \\
q^{k} \gamma, q b d e / \alpha \beta
\end{array} \mid q ; q\right] \\
& \quad+\left[\begin{array}{c}
\gamma / c, b d / \beta, q^{k} e, q^{k} \alpha \beta \gamma / b d e \\
q^{k} \alpha, q^{k} \gamma, b d e / \alpha \beta, \alpha \beta \gamma / b c d e
\end{array} \mid q\right]_{\infty}{ }_{3} \phi_{2}\left[\begin{array}{c}
\alpha / e, q^{k} \alpha \beta / b d, \alpha \beta \gamma / b c d e \\
q^{k} \alpha \beta \gamma / b d e, q \alpha \beta / b d e
\end{array} \mid q ; q\right] .
\end{aligned}
$$

Substituting this into (4) results in the first transformation formula.

Theorem 9 (Double sum expression).

$$
\begin{aligned}
& { }_{4} \phi_{3}\left[\begin{array}{c}
b, c, d, e \\
\alpha, \beta, \gamma
\end{array} \mid q ; \frac{\alpha \beta \gamma}{b c d e}\right]=\left[\begin{array}{c}
\alpha / e, \alpha \beta / b d \\
\alpha, \alpha \beta / b d e
\end{array} \mid q\right]_{\infty} \sum_{k, i \geq 0} \frac{(e ; q)_{k+i}}{(\gamma ; q)_{k+i}}\left(\frac{\alpha \gamma}{c e}\right)^{k} \\
& \times\left[\begin{array}{c}
c, \beta / b, \beta / d \\
q, \beta, \alpha \beta / b d
\end{array} \mid q\right]_{k}\left[\begin{array}{c}
\gamma / c, b d / \beta \\
q, q b d e / \alpha \beta
\end{array} \mid q\right]_{i} q^{i}+\left[\begin{array}{c}
e, \gamma / c, b d / \beta, \alpha \beta \gamma / b d e \\
\alpha, \gamma, b d e / \alpha \beta, \alpha \beta \gamma / b c d e
\end{array} \mid q\right]_{\infty} \\
& \times \sum_{k, i \geq 0} \frac{(\alpha \beta / b d ; q)_{k+i}}{(\alpha \beta \gamma / b d e ; q)_{k+i}}\left[\begin{array}{c}
c, \beta / b, \beta / d \\
q, \beta, \alpha \beta / b d
\end{array} \mid q\right]_{k}\left(\frac{\alpha \gamma}{c e}\right)^{k}\left[\begin{array}{c}
\alpha / e, \alpha \beta \gamma / b c d e \\
q, q \alpha \beta / b d e
\end{array} \mid q\right]_{i} q^{i}
\end{aligned}
$$

When $d=1$, the last theorem yields the following two-term relation.

Corollary 10 (Two-term summation formula).

$$
\begin{aligned}
& \frac{(\alpha \beta \gamma / b c e ; q)_{\infty}}{(\alpha \beta \gamma / b e ; q)_{\infty}}=\left[\begin{array}{c}
\alpha / e, \alpha \beta / b, \alpha \beta \gamma / b c e \\
\alpha, \alpha \beta / b e, \alpha \beta \gamma / b e
\end{array} \mid q\right]_{\infty} \\
& \times \sum_{k, i \geq 0} \frac{(e ; q)_{k+i}}{(\gamma ; q)_{k+i}}\left(\frac{\alpha \gamma}{c e}\right)^{k}\left[\left.\begin{array}{c}
c, \beta / b \\
q, \alpha \beta / b
\end{array}\right|_{k}\right]_{k}\left[\begin{array}{c}
\gamma / c, b / \beta \\
q, q b e / \alpha \beta
\end{array} \mid q\right]_{i} q^{i}+\left[\left.\begin{array}{c}
e, \gamma / c, b / \beta \\
\alpha, \gamma, b e / \alpha \beta
\end{array}\right|_{\infty}\right.
\end{aligned}
$$




$$
\times \sum_{k, i \geq 0} \frac{(\alpha \beta / b ; q)_{k+i}}{(\alpha \beta \gamma / b e ; q)_{k+i}}\left[\begin{array}{c}
c, \beta / b \\
q, \alpha \beta / b
\end{array} \mid q\right]_{k}\left(\frac{\alpha \gamma}{c e}\right)^{k}\left[\begin{array}{c}
\alpha / e, \alpha \beta \gamma / b c e \\
q, q \alpha \beta / b e
\end{array} \mid q\right]_{i} q^{i}
$$

3.2. By means of (9), the ${ }_{3} \phi_{2}$-series displayed in (4) can be expressed as

$$
\begin{aligned}
& { }_{3} \phi_{2}\left[\begin{array}{c}
q^{k} c, q^{k} e, b d / \beta \mid q ; \frac{\alpha \beta \gamma}{b c d e} \\
q^{k} \alpha, q^{k} \gamma
\end{array}\right]=\left[\begin{array}{c}
\alpha / c, \alpha / e \\
q^{k} \alpha, q^{-k} \alpha / c e
\end{array} \mid q\right]_{\infty} \\
& \times{ }_{3} \phi_{2}\left[\begin{array}{c}
q^{k} c, q^{k} e, q^{k} \beta \gamma / b d \\
q^{1+k} c e / \alpha, q^{k} \gamma
\end{array} \mid q ; q\right]+\left[\begin{array}{c}
q^{k} c, q^{k} e, q^{k} \beta \gamma / b d, \alpha \gamma / c e \\
q^{k} \alpha, q^{k} \gamma, q^{k} c e / \alpha, \alpha \beta \gamma / b c d e
\end{array} \mid q\right]_{\infty} \\
& \times{ }_{3} \phi_{2}\left[\begin{array}{c}
\alpha / c, \alpha / e, \alpha \beta \gamma / b c d e \\
\alpha \gamma / c e, q^{1-k} \alpha / c e
\end{array} \mid q ; q\right] .
\end{aligned}
$$

The corresponding (4) yields the second transformation formula.

Theorem 11 (Double sum expression).

$$
\begin{aligned}
& { }_{4} \phi_{3}\left[\begin{array}{c}
b, c, d, e \\
\alpha, \beta, \gamma
\end{array} \mid q ; \frac{\alpha \beta \gamma}{b c d e}\right]=\left[\begin{array}{c}
\alpha / c, \alpha / e \\
\alpha, \alpha / c e
\end{array} \mid q\right]_{\infty} \sum_{k, i \geq 0}\left[\begin{array}{c}
c, e, \beta \gamma / b d \\
q c e / \alpha, \gamma
\end{array} \mid q\right]_{k+i} \\
& \times\left[\begin{array}{c}
\beta / b, \beta / d \\
q, \beta, \beta \gamma / b d
\end{array} \mid q\right]_{k} \frac{q^{\left(\begin{array}{c}
k \\
2
\end{array}\right)+i}(-q \gamma)^{k}}{(q ; q)_{i}}+\left[\begin{array}{c}
c, e, \alpha \gamma / c e, \beta \gamma / b d \\
\alpha, \gamma, c e / \alpha, \alpha \beta \gamma / b c d e
\end{array} \mid q\right]_{\infty} \\
& \times \sum_{k, i \geq 0} \frac{q^{\left(\begin{array}{c}
k \\
2
\end{array}\right)+i}(-\gamma)^{k}}{(q \alpha / c e ; q)_{i-k}}\left[\begin{array}{c}
\beta / b, \beta / d \\
q, \beta, \beta \gamma / b d
\end{array} \mid q\right]_{k}\left[\begin{array}{c}
\alpha / c, \alpha / e, \alpha \beta \gamma / b c d e \\
q, \alpha \gamma / c e
\end{array} \mid q\right]_{i} .
\end{aligned}
$$

When $d=1$, the last theorem gives another two-term relation.

Corollary 12 (Two-term summation formula).

$$
\begin{aligned}
& \frac{(\alpha \beta \gamma / b c e ; q)_{\infty}}{(\alpha \gamma / c e ; q)_{\infty}} \\
= & {\left[\begin{array}{c}
\alpha / c, \alpha / e, \alpha \beta \gamma / b c e \\
\alpha, \alpha / c e, \alpha \gamma / c e
\end{array} \mid q\right]_{\infty} \sum_{k, i \geq 0}\left[\begin{array}{c}
c, e, \beta \gamma / b \\
q c e / \alpha, \gamma
\end{array} \mid q\right]_{k+i}\left[\begin{array}{c}
\beta / b \\
q, \beta \gamma / b
\end{array} \mid q\right]_{k} \frac{q^{\left(\begin{array}{c}
k \\
2
\end{array}\right)+i}(-q \gamma)^{k}}{(q ; q)_{i}} } \\
+ & {\left[\begin{array}{c}
c, e, \beta \gamma / b \\
\alpha, \gamma, c e / \alpha
\end{array} \mid q\right]_{\infty} \sum_{k, i \geq 0} \frac{q^{\left(\begin{array}{c}
k \\
2
\end{array}\right)+i}(-\gamma)^{k}}{(q \alpha / c e ; q)_{i-k}}\left[\begin{array}{c}
\beta / b \\
q, \beta \gamma / b
\end{array} \mid q\right]_{k}\left[\begin{array}{c}
\alpha / c, \alpha / e, \alpha \beta \gamma / b c e \\
q, \alpha \gamma / c e
\end{array} \mid q\right]_{i} . }
\end{aligned}
$$

3.3. Applying (10) to the ${ }_{3} \phi_{2}$-series in (4), we get the expression

$$
\begin{aligned}
& { }_{3} \phi_{2}\left[\begin{array}{c}
q^{k} c, q^{k} e, b d / \beta \\
q^{k} \alpha, q^{k} \gamma
\end{array} \mid q ; \frac{\alpha \beta \gamma}{b c d e}\right]={ }_{3} \phi_{2}\left[\begin{array}{c}
q^{k} e, q^{k} \alpha \beta / b d, q e / \gamma \\
q^{1+k} e \beta / b d, q^{1+k} c e / \gamma
\end{array} \mid q ; \frac{q c}{\alpha}\right] \\
& \left.\quad \times\left[\begin{array}{c}
\gamma / c, \gamma / e, q^{1-k} / \alpha, q^{1+k} e \beta / b d \\
q e / \alpha, q \beta / b d, q^{k} \gamma, q^{-k} \gamma / c e
\end{array} \mid q\right]_{\infty}\right]_{3} \phi_{2}\left[\begin{array}{c}
q c / \alpha, q e / \alpha, q^{1-k} b d / \alpha \beta \\
q \gamma / \alpha, q^{2-k} / \alpha
\end{array} \mid q ; \frac{\alpha \beta \gamma}{b c d e}\right]
\end{aligned}
$$




$$
\times\left[\begin{array}{l}
q \gamma / \alpha, q^{1-k} / \alpha, q^{k} c, q^{k} e, q^{k} \alpha \beta / b d, \alpha \gamma / q c e, q^{2} c e / \alpha \gamma \\
q^{k-1} \alpha, q^{k} \gamma, q c / \alpha, q e / \alpha, q \beta / b d, q^{-k} \gamma / c e, q^{1+k} c e / \gamma
\end{array}\right]_{\infty} .
$$

Substituting the last equation into (4) gives rise to the third transformation formula.

Theorem 13 (Double sum expression).

$$
\begin{aligned}
& { }_{4} \phi_{3}\left[\begin{array}{r}
\left.b, c, d, e \mid q ; \frac{\alpha \beta \gamma}{b c d e}\right] \\
\alpha, \beta, \gamma
\end{array}\right]=\left[\begin{array}{c}
q / \alpha, \gamma / c, \gamma / e, q \beta e / b d \\
\gamma, \gamma / c e, q e / \alpha, q \beta / b d
\end{array} \mid q\right]_{\infty} \\
& \times \sum_{k, i \geq 0} q^{k}\left[\begin{array}{c}
e, \alpha \beta / b d \\
q e \beta / b d, q c e / \gamma
\end{array} \mid q\right]_{k+i}\left[\begin{array}{c}
c, \beta / b, \beta / d \\
q, \beta, \alpha \beta / b d
\end{array} \mid q\right]_{k} \frac{(q e / \gamma ; q)_{i}}{(q ; q)_{i}}\left(\frac{q c}{\alpha}\right)^{i} \\
& +\frac{q}{\alpha}\left[\begin{array}{c}
q^{2} / \alpha, q \gamma / \alpha, c, e, \alpha \beta / b d, \alpha \gamma / q c e, q^{2} c e / \alpha \gamma \\
\alpha, \gamma, q c / \alpha, q e / \alpha, q \beta / b d, \gamma / c e, q c e / \gamma
\end{array} \mid q\right]_{\infty} \\
& \times \sum_{k, i \geq 0} q^{k} \frac{(\alpha / q ; q)_{k-i}}{(\alpha \beta / b d ; q)_{k-i}}\left[\begin{array}{c}
\beta / b, \beta / d \\
q, \beta
\end{array} \mid q\right]_{k}\left[\begin{array}{c}
q c / \alpha, q e / \alpha \\
q, q \gamma / \alpha
\end{array} \mid q\right]_{i}\left(\frac{\alpha \gamma}{q c e}\right)^{i} .
\end{aligned}
$$

For this theorem and the next one, we shall not produce the two-term relations corresponding to the case $d=1$, which will resemble Corollaries 10 and 12 .

3.4. Finally, the ${ }_{3} \phi_{2}$-series displayed in (4) can be reformulated through (10) as

$$
\begin{aligned}
& { }_{3} \phi_{2}\left[\begin{array}{c}
q^{k} c, q^{k} e, b d / \beta \mid q ; \frac{\alpha \beta \gamma}{b c d e} \\
q^{k} \alpha, q^{k} \gamma
\end{array}\right]=\left[\begin{array}{c}
\gamma / e, q^{k} \beta \gamma / b d, q^{1-k} / \alpha, q^{1-k} b d / c \beta \\
\beta \gamma / b d e, q^{1-k} / c, q^{k} \gamma, q^{1-k} b d / \alpha \beta
\end{array} \mid q\right]_{\infty} \\
& \times{ }_{3} \phi_{2}\left[\begin{array}{c}
b d / \beta, \alpha / c, q^{1-k} b d / \beta \gamma \\
q b d e / \beta \gamma, q^{1-k} b d / c \beta
\end{array} \mid q ; \frac{q e}{\alpha}\right]-{ }_{3} \phi_{2}\left[\begin{array}{c}
\frac{q c}{\alpha}, \frac{q e}{\alpha}, \frac{q^{1-k} b d}{\alpha \beta} \mid q \gamma / \alpha, q^{2-k} / \alpha \\
q \gamma / \alpha \gamma
\end{array}\right] \\
& \times\left[\begin{array}{c}
q \gamma / \alpha, q^{1-k} / \alpha, q^{k} e, b d / \beta, \alpha / c, q^{k-1} \alpha \beta \gamma / b d e, q^{2-k} b d e / \alpha \beta \gamma \\
q^{k-1} \alpha, q^{k} \gamma, q e / \alpha, \beta \gamma / b d e, q^{1-k} b d / \alpha \beta, q^{1-k} / c, q b d e / \beta \gamma
\end{array} \mid q\right]_{\infty}
\end{aligned}
$$

which leads (4) to the fourth transformation formula.

Theorem 14 (Double sum expression).

$$
\begin{aligned}
& { }_{4} \phi_{3}\left[\begin{array}{c}
\left.b, c, d, e \mid q ; \frac{\alpha \beta \gamma}{b c d e}\right] \\
\alpha, \beta, \gamma
\end{array}\right]=\left[\begin{array}{c}
\gamma / e, \beta \gamma / b d, q b d / c \beta, q / \alpha \\
\gamma, q b d / \alpha \beta, q / c, \beta \gamma / b d e
\end{array} \mid q\right]_{\infty} \\
& \times \sum_{k, i \geq 0} \frac{(c \beta / b d ; q)_{k-i}}{(\beta \gamma / b d ; q)_{k-i}}\left[\begin{array}{c}
e, \beta / b, \beta / d \\
q, \beta, \alpha \beta / b d
\end{array} \mid q\right]_{k}\left(\frac{\alpha \gamma}{c e}\right)^{k}\left[\begin{array}{c}
\alpha / c, b d / \beta \\
q, q b d e / \beta \gamma
\end{array} \mid q\right]_{i}\left(\frac{q c e}{\alpha \gamma}\right)^{i} \\
& +\frac{q}{\alpha}\left[\begin{array}{c}
q^{2} / \alpha, q \gamma / \alpha, e, b d / \beta, \alpha / c, \alpha \beta \gamma / b d e q, q^{2} b d e / \alpha \beta \gamma \\
\alpha, \gamma, q b d / \alpha \beta, q e / \alpha, q / c, \beta \gamma / b d e, q b d e / \beta \gamma
\end{array} \mid q\right]_{\infty}
\end{aligned}
$$




$$
\times \sum_{k, i \geq 0} q^{k} \frac{(\alpha / q ; q)_{k-i}}{(\alpha \beta / b d ; q)_{k-i}}\left[\begin{array}{c}
\beta / b, \beta / d \\
q, \beta
\end{array} \mid q\right]_{k}\left[\begin{array}{c}
q c / \alpha, q e / \alpha \\
q, q \gamma / \alpha
\end{array} \mid q\right]_{i}\left(\frac{\alpha \gamma}{q c e}\right)^{i}
$$

It is widely known that some two-term relations can be rewritten in compact form in terms of $q$-integrals. For the $q$-double series, there is also such an example in Gasper-Rahman [4, Exercise 10.16]). However, it is unlikely to do so for the theorems derived in this section because the two double sums involved are very different in their structure. The only exception is Theorem 9 with the two double sums having the same symmetric form. However, it seems an uneasy task to express it in $q$-integrals due to the presence of "variable" $(\alpha \gamma / c e)^{k}$.

\section{Further KNOWN EXAMPLES}

The four theorems established in the last section are all reduced to the Sears transformation (1) when the ${ }_{4} \phi_{3}$-series is terminating balanced one. In fact, for $\alpha \beta \gamma=q b c d e$, the ${ }_{4} \phi_{3}$-series in Theorem 9 is balanced. The corresponding result can be stated as the following proposition.

Proposition $15(\alpha \beta \gamma=q b c d e)$.

$$
\begin{aligned}
& { }_{4} \phi_{3}\left[\begin{array}{c}
b, c, d, e \\
\alpha, \beta, \gamma
\end{array} \mid q ; q\right]=\left[\begin{array}{c}
\alpha / e, \alpha \beta / b d \\
\alpha, \alpha \beta / b d e
\end{array} \mid q\right]_{\infty} \sum_{k, i \geq 0} \frac{(e ; q)_{k+i}}{(\gamma ; q)_{k+i}}\left(\frac{\alpha \gamma}{c e}\right)^{k} \\
& \times\left[\begin{array}{c}
c, \beta / b, \beta / d \\
q, \beta, \alpha \beta / b d
\end{array} \mid q\right]_{k} \frac{(b d / \beta ; q)_{i}}{(q ; q)_{i}} q^{i}-\frac{q c}{\gamma}\left[\begin{array}{c}
e, q c, b d / \beta \\
q, \alpha, \gamma
\end{array} \mid q\right]_{\infty} \\
& \times \sum_{k, i \geq 0}\left(\frac{\alpha \gamma}{c e}\right)^{k}\left[\begin{array}{c}
c, \beta / b, \beta / d \\
q, \beta, \alpha \beta / b d
\end{array} \mid q\right]_{k} \frac{(\alpha \beta / b d ; q)_{k+i}(\alpha / e ; q)_{i}}{(q c ; q)_{k+i}(\alpha \beta / b d e ; q)_{i+1}} q^{i}
\end{aligned}
$$

When $e=q^{-n}$, the second part will be annihilated by the zero factor $\left(q^{-n} ; q\right)_{\infty}$, while the first sum can be manipulated as follows

$$
\begin{aligned}
\sum_{k, i \geq 0} \frac{\left(q^{-n} ; q\right)_{k+i}}{(\gamma ; q)_{k+i}}\left(\frac{\alpha \gamma}{c}\right)^{k}\left[\begin{array}{c}
c, \beta / b, \beta / d \\
q, \beta, \alpha \beta / b d
\end{array} \mid q\right]_{k} \frac{(b d / \beta ; q)_{i}}{(q ; q)_{i}} q^{i+k n} \\
=\sum_{k \geq 0} q^{k n}\left[\begin{array}{c}
q^{-n}, c, \beta / b, \beta / d \\
q, \beta, \gamma, \alpha \beta / b d
\end{array} \mid q\right]_{k}\left(\frac{\alpha \gamma}{c}\right)^{k}{ }_{2} \phi_{1}\left[\begin{array}{c}
q^{k-n}, b d / \beta \\
q^{k} \gamma
\end{array} \mid q ; q\right] .
\end{aligned}
$$

Evaluating the ${ }_{2} \phi_{1}$-series by means of the $q$-Chu-Vandermonde-Gauss formula (cf. $[4, \mathrm{II}-6])$

$$
\begin{aligned}
{ }_{2} \phi_{1}\left[\begin{array}{c}
q^{k-n}, b d / \beta \\
q^{k} \gamma
\end{array} \mid q ; q\right] & =\frac{\left(q^{k} \beta \gamma / b d ; q\right)_{n-k}}{\left(q^{k} \gamma ; q\right)_{n-k}}\left(\frac{b d}{\beta}\right)^{n-k} \\
& =\frac{(\beta \gamma / b d ; q)_{n}(\gamma ; q)_{k}}{(\beta \gamma / b d ; q)_{k}(\gamma ; q)_{n}}\left(\frac{b d}{\beta}\right)^{n-k}
\end{aligned}
$$


we find that the last double sum reduces to a single one. Then the resulting expression gives the Sears transformation in (1) after some routine simplification.

Two further particular cases of Proposition 15 may be worthy of mentioning. The first case $\alpha=e$ results in a special form of (5) when the series is balanced. Another one is the nonterminating $q$-Vandermonde sum (cf. [4, II-23]) which corresponds to the case $c=1$.

Corollary $16(\alpha \beta \gamma=q b d e)$.

${ }_{2} \phi_{1}\left[\begin{array}{r}a, b \\ c \mid\end{array} \mid q ; q\right]=\left[\begin{array}{l}q / c, q a b / c \\ q a / c, q b / c \mid\end{array} \mid q\right]_{\infty}+\frac{q}{c}\left[\begin{array}{c}q^{2} / c, a, b \\ c, q a / c, q b / c\end{array} \mid q\right]_{\infty}{ }_{2} \phi_{1}\left[\begin{array}{r}q a / c, q b / c \\ q^{2} / c\end{array} \mid q ; q\right]$.

Analogously, we may examine Theorem 13. When $\alpha \beta \gamma=q b c d e$, the ${ }_{4} \phi_{3^{-}}$ series is balanced. Then the first double sum can be reduced to a single one

$$
\begin{aligned}
& \sum_{k, i \geq 0} q^{k}\left[\begin{array}{c}
e, \alpha \beta / b d \\
q e \beta / b d, q c e / \gamma
\end{array} \mid q\right]_{k+i} \frac{(q e / \gamma ; q)_{i}}{(q ; q)_{i}}\left[\begin{array}{c}
c, \beta / b, \beta / d \\
q, \beta, \alpha \beta / b d
\end{array} \mid q\right]_{k}\left(\frac{q c}{\alpha}\right)^{i} \\
& =\sum_{k \geq 0} q^{k}\left[\begin{array}{c}
c, e, \beta / b, \beta / d \\
q, \beta, \alpha \beta / b d, q e \beta / b d
\end{array} \mid q\right]_{k}{ }_{2} \phi_{1}\left[\begin{array}{c}
q e / \gamma, q^{k} e \\
q^{1+k} e \beta / b d
\end{array} \mid q ; \frac{q c}{\alpha}\right] \\
& =\sum_{k \geq 0} q^{k}\left[\begin{array}{c}
c, e, \beta / b, \beta / d \\
q, \beta, \alpha \beta / b d, q \beta e / b d
\end{array} \mid q\right]_{k}\left[\begin{array}{c}
q^{k} \beta \gamma / b d, q \beta / b d \\
q^{1+k} e \beta / b d, q c / \alpha
\end{array} \mid q\right]_{\infty} \\
& =\left[\begin{array}{l}
\beta \gamma / b d, q \beta / b d \\
q e \beta / b d, q c / \alpha
\end{array} \mid q\right]_{\infty}{ }_{4} \phi_{3}\left[\begin{array}{c}
c, e, \beta / b, \beta / d \\
q, \beta, \alpha \beta / b d, \beta \gamma / b d
\end{array} \mid q ; q\right]
\end{aligned}
$$

where the last ${ }_{2} \phi_{1}$-series has been evaluated through (7). Hence, Theorem 13 under the balanced condition $\alpha \beta \gamma=q b c d e$ becomes the following transformation formula.

Proposition $17(\alpha \beta \gamma=q b c d e)$.

$$
\begin{aligned}
{ }_{4} \phi_{3}\left[\begin{array}{c}
b, c, d, e \mid \\
\alpha, \beta, \gamma
\end{array} \mid q ; q\right] & =\left[\begin{array}{c}
q / \alpha, \gamma / c, \gamma / e, q c e / \alpha \\
\gamma, q c / \alpha, q e / \alpha, \gamma / c e
\end{array} \mid q\right]_{\infty}{ }_{4} \phi_{3}\left[\begin{array}{c}
c, e, \beta / b, \beta / d \\
\beta, \alpha \beta / b d, \beta \gamma / b d
\end{array} \mid q ; q\right] \\
& +\frac{q}{\alpha}\left[\begin{array}{c}
q^{2} / \alpha, c, e, b d / \beta, q \gamma / \alpha \\
\alpha, q c / \alpha, q e / \alpha, q b d / \alpha \beta, \gamma
\end{array} \mid q\right]_{\infty} \\
& \times \sum_{k, i \geq 0} q^{k}\left[\begin{array}{c}
\beta / b, \beta / d \\
q, \beta
\end{array} \mid q\right]_{k} \frac{(\alpha / q)_{k-i}}{(\alpha \beta / b d)_{k-i}}\left[\begin{array}{c}
q c / \alpha, q e / \alpha \\
q, q \gamma / \alpha
\end{array} \mid q\right]_{i}\left(\frac{b d}{\beta}\right)^{i} .
\end{aligned}
$$

Furthermore, we can check without difficulty that the terminating case $e=q^{-n}$ of this proposition recovers again the Sears transformation (1). Instead, when $\beta=d$, Proposition 17 reduces to the following nonterminating $q$-Pfaff-Saalschütz theorem (cf. [4, II-24]). 
Corollary $18(\alpha \gamma=q b c d)$.

$$
\begin{aligned}
{ }_{3} \phi_{2}\left[\begin{array}{r}
b, c, d \\
\alpha, \gamma
\end{array} \mid q ; q\right] & =\left[\begin{array}{c}
q / \gamma, \alpha / b, \alpha / c, \alpha / d \\
\alpha, q b / \gamma, q c / \gamma, q d / \gamma
\end{array} \mid q\right] \\
+ & \frac{q}{\gamma}\left[\begin{array}{c}
q \alpha / \gamma, q^{2} / \gamma, b, c, d \\
\alpha, \gamma, q b / \gamma, q c / \gamma, q d / \gamma
\end{array} \mid q\right]_{\infty}{ }_{\infty} \phi_{2}\left[\begin{array}{c}
q b / \gamma, q c / \gamma, q d / \gamma \\
q^{2} / \gamma, q \alpha / \gamma
\end{array} \mid q ; q\right] .
\end{aligned}
$$

Before ending the paper, we would like to point out that all the theorems obtained in this paper involve the double $q$-series. One important class of them is called $q$-Kampé de Fériet Series (cf. Gasper-Rahman [4, §10.2] for example). The informed reader can check without difficulty that Theorem 3 is, in fact, equivalent to a transformation due to Chu-Jia [2, Proposition 2.3], while Theorem 7 is essentially a reduction formula. Further summation and transformation formulae can be found in the papers by Chu et al $[2,3]$.

Acknowledgement. The authors are sincerely grateful to an anonymous referee for the critical comments and valuable suggestions, that helped us substantially to improve the manuscript in revision.

\section{REFERENCES}

[1] W. N. Bailey, Generalized Hypergeometric Series, Cambridge University Press, Cambridge, 1935.

[2] W. Chu and C. Jia, Transformation and reduction formulae for double q-Clausen hypergeometric series, Math. Methods Appl. Sci., 31 (1) (2008), 1-17.

[3] W. Chu and N. N. Li, Terminating $q$-Kampé de Fériet series $\Phi_{1: 2 ; \mu}^{1: 3 ; \lambda}$ and $\Phi_{2: 1 ; \mu}^{2: 2 ; \lambda}$, Hiroshima Math. J., 42 (2) (2012), 233-252.

[4] G. Gasper and M. Rahman, Basic Hypergeometric Series (2nd ed.), Cambridge University Press, Cambridge, 2004.

[5] D. B. Sears, On the transformation theory of basic hypergeometric functions, Proc. London Math. Soc., 53 (1951), 158-180.

(Received: August 30, 2015)

(Revised: January 1, 2016)

\author{
Wenchang Chu \\ Dipartimento di Matematica e Fisica \\ "Ennio De Giorgi" \\ Universitá del Salento \\ Lecce-Arnesano P. O. Box 193 \\ 73100 Lecce \\ Italy \\ chu.wenchang@unisalento.it \\ Nadia N. Li \\ Department of Mathematics \\ Zhoukou Normal University \\ Zhoukou 466000 \\ P. R. China \\ lina3718@163.com
}


